\title{
Small-Molecule Screen in Adult Drosophila Identifies VMAT as a Regulator of Sleep
}

\author{
Aleksandra H. Nall ${ }^{1}$ and Amita Sehgal ${ }^{1,2}$ \\ ${ }^{1}$ Cell and Molecular Biology Program and ${ }^{2}$ Department of Neuroscience, Howard Hughes Medical Institute, Perelman School of Medicine, University of \\ Pennsylvania, Philadelphia, Pennsylvania 19104
}

Sleep is an important physiological state, but its function and regulation remain elusive. In Drosophila melanogaster, a useful model organism for studying sleep, forward genetic screens have identified important sleep-modulating genes and pathways; however, the results of such screens may be limited by developmental abnormalities or lethality associated with mutation of certain genes. To circumvent these limitations, we used a small-molecule screen to identify sleep-modulating genes and pathways. We administered 1280 pharmacologically active small molecules to adult flies and monitored their sleep. We found that administration of reserpine, a smallmolecule inhibitor of the vesicular monoamine transporter (VMAT) that repackages monoamines into presynaptic vesicles, resulted in an increase in sleep. Supporting the idea that VMAT is the sleep-relevant target of reserpine, we found that VMAT-null mutants have an increased sleep phenotype, as well as an increased arousal threshold and resistance to the effects of reserpine. However, although the VMAT mutants are consistently resistant to reserpine, other aspects of their sleep phenotype are dependent on genetic background. These findings indicate that small-molecule screens can be used effectively to identify sleep-modulating genes whose phenotypes may be suppressed in traditional genetic screens. Mutations affecting single monoamine pathways did not affect reserpine sensitivity, suggesting that effects of VMAT/reserpine on sleep are mediated by multiple monoamines. Overall, we identify VMAT as an important regulator of sleep in Drosophila and demonstrate that small-molecule screens provide an effective approach to identify genes and pathways that impact adult Drosophila behavior.

\section{Introduction}

Sleep is an important physiological state, as evidenced by the fact that we spend a third of our lives in this state. Additionally, sleep deprivation causes cognitive and health deficits, indicating that it plays an essential role in physiological homeostasis. Sleep is not just required in humans; all well-studied animals exhibit sleeplike states. Among these animals are common model organisms, including mice, zebrafish, flies, and nematode worms (Allada and Siegel, 2008; Mackiewicz et al., 2008; Raizen et al., 2008; Bushey and Cirelli, 2011). These models and others are being used to investigate outstanding questions regarding the purpose of sleep and its regulation.

The rest state in the fly shares many commonalities with human sleep behavior (Shaw et al., 2000, Hendricks et al., 2000). For example, sleeping flies stop moving and assume a stereotyped posture. They also exhibit an increased arousal threshold, meaning that they require a stronger stimulus to reinitiate activity.

\footnotetext{
Received Jan. 18, 2013; revised April 2, 2013; accepted April 10, 2013.

Author contributions: A.H.N. and A.S. designed research; A.H.N. performed research; A.H.N. analyzed data; A.H.N. and A.S. wrote the paper.

This work was supported by the National Institutes of Health Grants 5 T32 HL 7953-12 and 5-P01-AG-017628. We thank Dr. John Hogenesch (University of Pennsylvania, Philadelphia) for the small-molecule library and Dr. David Krantz (UCLA, Los Angeles) and Dr. Ralph Hillman (New York University, New York) for fly lines.

The authors declare no competing financial interests.

Correspondence should be addressed to Dr. Amita Sehgal, Department of Neuroscience, Howard Hughes Medical Institute, University of Pennsylvania School of Medicine, 3620 Hamilton Walk, Philadelphia, PA 19104. E-mail: amita@mail.med.upenn.edu.

DOI:10.1523/JNEUROSCI.0253-13.2013

Copyright $\odot 2013$ the authors $\quad 0270-6474 / 13 / 338534-07 \$ 15.00 / 0$
}

Importantly, flies display a homeostatic need for sleep, such that they compensate for periods of sleep deprivation with subsequent rebound. Drosophila melanogaster follow a diurnal pattern, resting mostly during the night and taking a mid-afternoon "siesta."

The simplicity of behavioral assays using Drosophila, combined with the ease of genetic screens, has led many researchers to turn to this model to elucidate the genetic and molecular mechanisms underlying sleep regulation. Genetic screens have uncovered a few low-sleeping mutants, but these studies have not yet led to a cohesive account of sleep regulation. Given that sleep appears to be an essential process (Rechtschaffen et al., 1983; Shaw et al., 2009), it is likely that many sleep-regulating genes are also essential. In other words, loss of these genes may cause lethality or gross developmental problems, precluding their detection in traditional genetic screens. To complement previous genetic screens and to find novel sleep-regulatory molecules and pathways, we conducted a small-molecule screen for sleep phenotypes in adult Drosophila. Here, we report the findings from this screen, which indicate a strong effect of monoaminergic neurotransmission in regulating sleep quantity. Using both pharmacological and genetic approaches, we investigated the role of one of our hits in regulating sleep behavior.

\section{Materials and Methods}

Flies. Wild-type iso31 flies (Ryder et al., 2004) were used for drug screen and subsequent experiments. VMAT ${ }^{p 1}$ mutants were a kind gift of the Krantz laboratory (University of California-Los Angeles, Los Angeles,

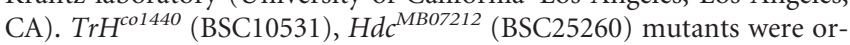



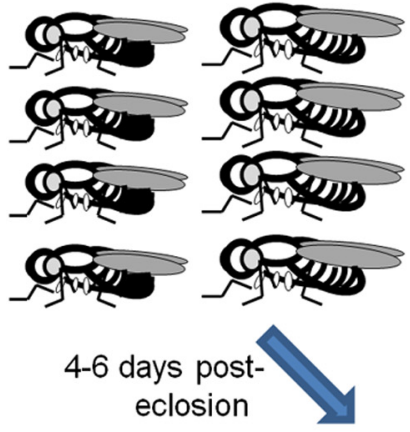

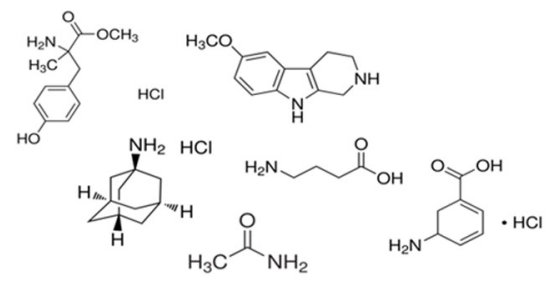

\section{0 known bioactives}

20uM
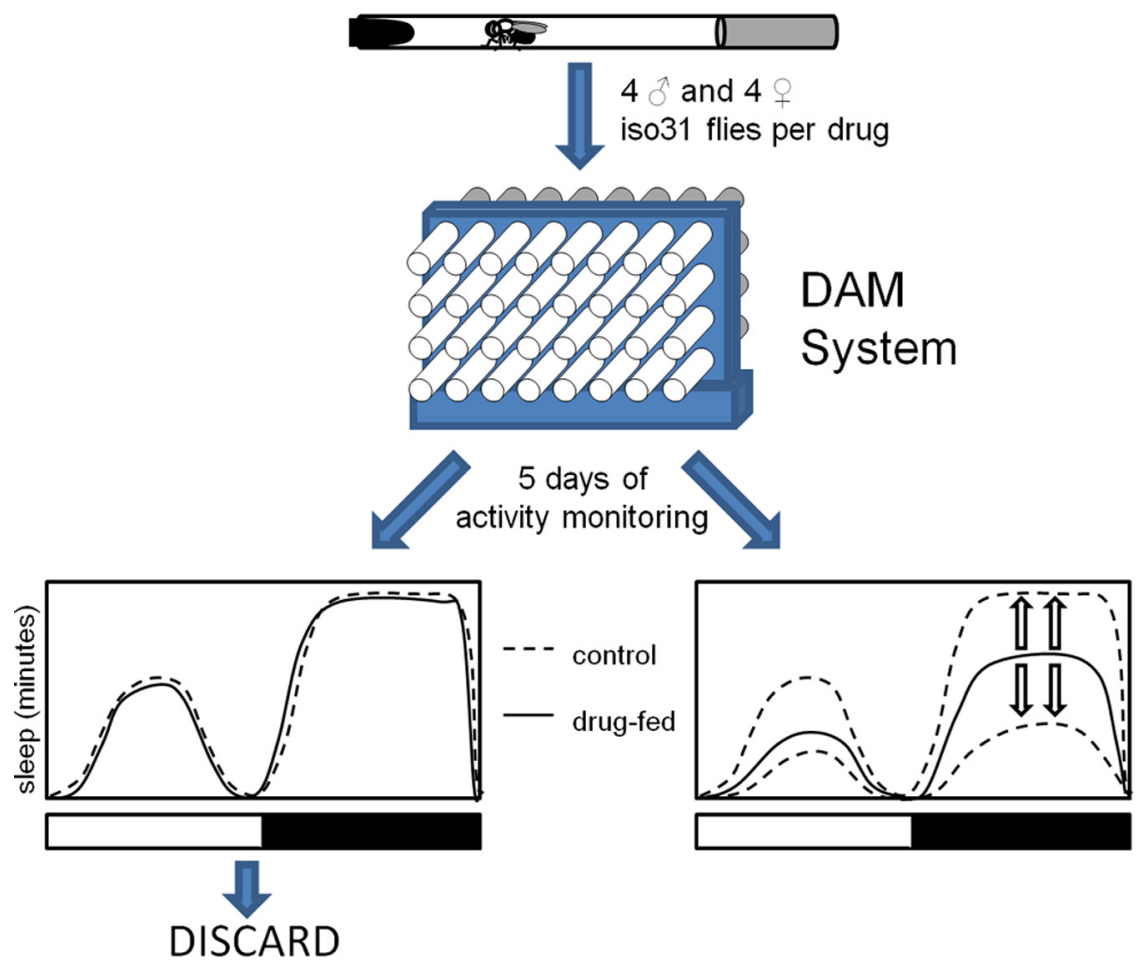

Re-test at 50uM

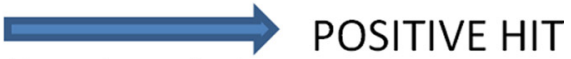

Dose-dependent

effect in both $\hat{\delta} ?$

POSITIVE HIT

Figure 1. Schematic of the small-molecule screen. Four male and 4 female flies were screened for each of 1280 known bioactive drugs. At $4-6 \mathrm{~d}$ after eclosion, adult flies were put in tubes with food containing $20 \mu \mathrm{m}$ of drug. These tubes were placed in monitors, and locomotor activity was measured for $5 \mathrm{~d}$ using the Drosophila Activity Monitoring (DAM) System. Using pySolo, sleep profiles were generated for males and females for each drug and compared with flies fed $0.01 \%$ DMSO (control). If a drug altered sleep in both males and females, it was retested at $50 \mu \mathrm{m}$. If the drug had a dose-dependent effect, it was considered a positive hit.

dered from the Bloomington Stock Center (Bloomington, IN), and Gad1 ${ }^{f 00602}$ was ordered from the Exelixis collection at Harvard Medical School. The temperature-sensitive tyrosine hydroxylase mutant $p l e^{t s}$ (Pendleton et al., 2002) was a kind gift from Dr. Ralph Hillman (New York University, NY). The octopamine synthesis mutant $T b H^{n m 18}$ was previously published (Crocker and Sehgal, 2008).

Drug feeding. We used the LOPAC 1280 drug library (Sigma-Aldrich), which is made up of bioactive molecules with known molecular targets, of which approximately half are involved in neurotransmission. Four- to 6 -day-old adult isogenic (iso31) flies were given access to drugs at $20 \mu \mathrm{M}$, mixed into their $2 \%$ agar and $5 \%$ sucrose food, ad libitum for 1 week. Flies were kept in incubators at $25^{\circ} \mathrm{C}$ on a $12 \mathrm{~h}$ light/dark schedule. During this time, locomotor activity of flies was monitored using the Drosophila Activity Monitoring System (Trikinetics). Sleep behavior was calculated and averaged for four male flies and four female flies per drug treatment. Sleep graphs and calculations of sleep quantity for all experiments were generated using PySolo (Gilestro and Cirelli, 2009). For drugs that produced qualitative changes in sleep profile or quantitative changes in total minutes of sleep per day, four male and four female flies were tested again at $50 \mu \mathrm{M}$ drug. Drugs that showed a reproducible, dose-dependent effect on sleep quantity were considered screen hits. For reserpine (Sigma-Aldrich), the stock solution was made at $10 \mathrm{~mm}$ in DMSO. This stock solution was diluted in $2 \%$ agar $/ 5 \%$ sucrose food to final concentrations of 20 and $50 \mu \mathrm{M}$ for the original screen and $10 \mu \mathrm{M}$ for all subsequent experiments. A total of $0.2 \%$ DMSO vehicle controls were used as a comparison for $20 \mu \mathrm{M}$ drug feeding during the screen, and $0.1 \%$ DMSO vehicle controls were used as a comparison for $10 \mu \mathrm{M}$ reserpine feeding in subsequent experiments. For the $p l e^{t s}$ mutants, flies were kept at a restrictive temperature of $29^{\circ} \mathrm{C}$ for $24 \mathrm{~h}$ before placing on DMSO and reserpine and were kept at this temperature for the duration of behavioral monitoring.

Arousal threshold. Arousal threshold assay was conducted as previously published (Wu et al., 2008). Mechanical stimuli were applied manually by tapping a dowel on the behavior tubes containing the flies. Weak (one light tap), medium (one strong tap), and strong (six strong taps) stimuli were applied to behavior tubes at ZT16, ZT18, and ZT20, respectively. The percentage of spontaneously sleeping flies awoken was calculated for each genotype and stimulus.

Sleep deprivation. Flies were deprived during the final $6 \mathrm{~h}$ of the night (ZT18-ZT24) using a vortex to shake flies for $2 \mathrm{~s}$ of every $20 \mathrm{~s}$, at random intervals (Huber et al., 2004). Amount of sleep lost was calculated by subtracting minutes of sleep during deprivation from the minutes of 
sleep during the same interval on the previous night. Sleep regained the following morning was calculated by subtracting the minutes of sleep during the first 3,6 , or $12 \mathrm{~h}$ the morning before deprivation from the same interval after deprivation.

PCR. The wild-type vesicular monoamine transporter (VMAT) allele was genotyped using VMATp1-F (5'-ATC GGG GGA TGC TTG ATA TT-3') and VMATp1-R (5'-ATC CGA ATC GGG AAC AGA T-3') primers, and the mutant $\mathrm{VMAT}^{\mathrm{p}}{ }^{1}$ allele was genotyped using the Plac1 $\left(5^{\prime}-\right.$ CAC CCA AGG CTC TGC TCC CAC AA-3') primer and VMATp1-R primers. PCR was conducted with GoTaq Flexi (Promega), with the following cycling conditions: $95^{\circ} \mathrm{C}$ for $2 \mathrm{~min}$, then 30 cycles of $95^{\circ} \mathrm{C}$ for $30 \mathrm{~s}, 52^{\circ} \mathrm{C}$ for $1 \mathrm{~min}, 72^{\circ} \mathrm{C}$ for $1 \mathrm{~min}$, and final extension at $72^{\circ} \mathrm{C}$ for $5 \mathrm{~min}$.

Sleep latency. Latency to sleep was calculated by counting the number of minutes between lights off and the first stretch of 5 consecutive minutes with zero beam crosses, as recorded by the Drosophila Activity Monitoring System.

\section{Results}

A small-molecule screen identifies

sleep-modulating compounds

We screened 1280 small molecules for their effect on sleep:wake rhythms in the adult fly (Fig. 1). Observation of daily locomotor behavior allowed for quantitative comparisons of total sleep time, daytime and nighttime sleep, and qualitative assessment of sleep patterns, rhythm strength, and anticipation of light/dark transitions. Each drug was assayed in a limited number of flies to enhance throughput; therefore, only drugs with strong effects on sleep were identified as having an effect above individual variation. Additionally, all compounds were fed to flies at a relatively low dose that caused minimal lethality. Many of these drugs showed an effect in only one sex and were discarded from further testing. Even with these constraints, we were able to identify 38 compounds that affected sleep at the initial concentration. Only those drugs found to have a dose-dependent effect on sleep in both sexes when tested at a higher concentration were considered hits. With these stringent criteria, we initially found six compounds that qualified: five that decreased sleep and one that increased sleep. One of the sleep-reducing compounds, the cholinergic agonist carbachol, did not continue to have an effect in subsequent studies (data not shown). The four remaining sleep-reducing drugs caused a significant reproducible decrease in nighttime sleep at a $20 \mu \mathrm{M}$ concentration and a further reduction in sleep levels at $50 \mu \mathrm{M}$. These sleep-promoting drugs are pergolide methanesulfonate (Fig. 2a), $\mathrm{R}(-)$-2,10,11-trihydroxyaporphine hydrobromide (Fig. 2b), paliperidone (Fig. 2c), and 1,3-dipropyl-7-methylxanthine (Fig. 2d). The screen revealed a single sleep-promoting drug, reserpine (Fig. $2 e$ ). Reserpine caused a significant increase in sleep during both the day and night, especially at light/dark transitions when flies are most active ( $p=0.000161$ by one-way ANOVA with Tukey post hoc comparison for both the $20 \mu \mathrm{M}$ and $50 \mu \mathrm{M}$ reserpine-fed flies compared with DMSO controls). In female flies, sleep increased by $400 \mathrm{~min}$ at $20 \mu \mathrm{M}$ reserpine and by $470 \mathrm{~min}$ at $50 \mu \mathrm{M}$ reserpine (Fig. $3 a$ ). Male flies showed a similar significant behavioral response to drug treatment (data not shown).

\section{Genetic ablation of VMAT alters sleep behavior}

Reserpine is an inhibitor of the VMAT. To determine whether the effects of reserpine on sleep were mediated by its inhibition of VMAT, we compared the sleep phenotype of reserpine-treated flies with that of a VMAT-null mutant, VMAT ${ }^{p 1}$ (Simon et al., 2009). The VMAT ${ }^{p 1}$ homozygous mutant has the same significantly increased sleep quantity as flies fed $50 \mu \mathrm{M}$ reserpine $(p=$ 0.000144 for $V M A T^{p 1} / V M A T^{p 1}$ compared with iso31 as shown by one-way ANOVA with Tukey post hoc comparison) (Fig. 3a).

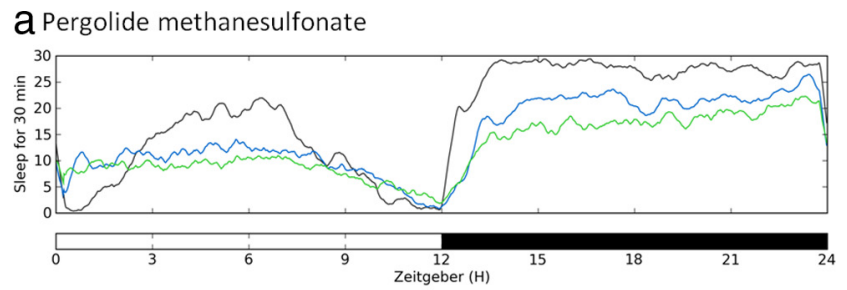

b R(-)-2,10,11-trihydroxyaporphine hydrobromide
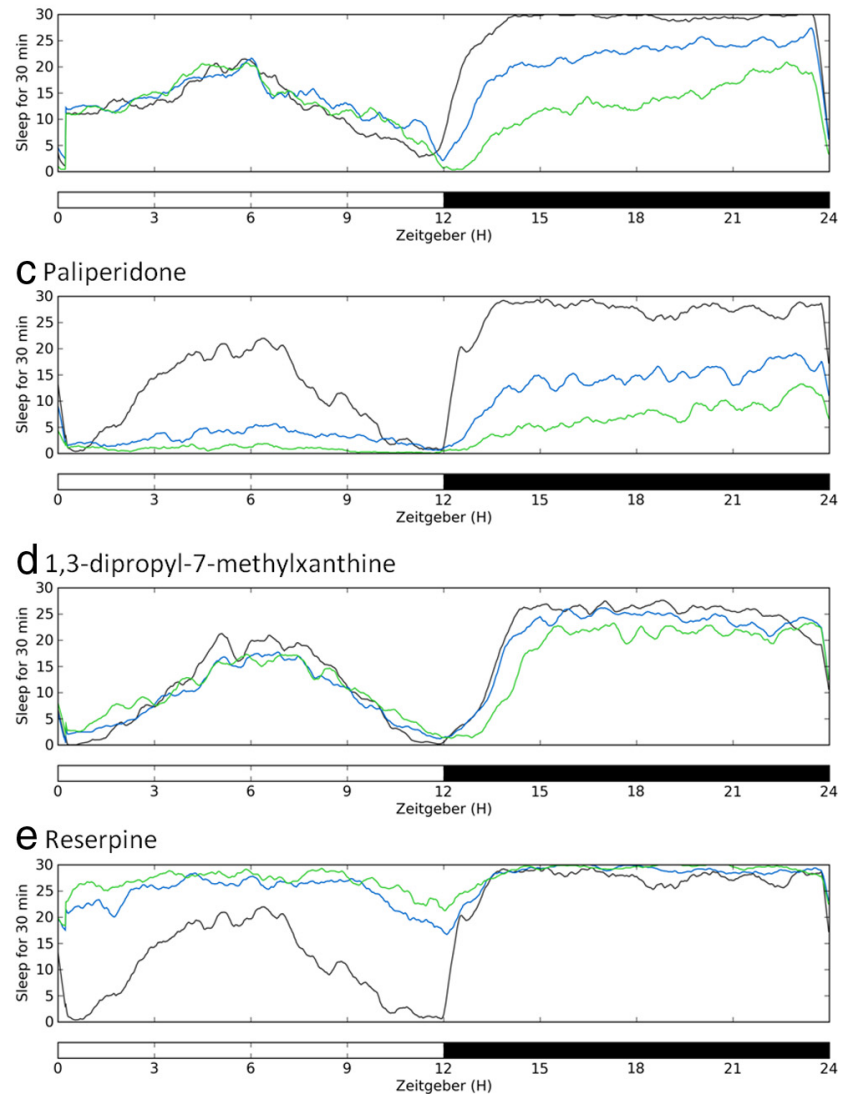

Figure 2. Small-molecule screen identifies sleep-modulating compounds. Sleep profiles for female flies fed 0.1\% DMSO (black line), $20 \mu \mathrm{m}$ drug (blue line), and $50 \mu \mathrm{m}$ drug (green line) for each of the positive hits from the screen: pergolide methanesulfonate $(\boldsymbol{a}), R(-)-2,10,11-$ trihydroxyaporphine hydrobromide $(\boldsymbol{b})$, paliperidone $(\boldsymbol{c})$, 1,3-dipropyl-7-methylxanthine $(\boldsymbol{d})$, and reserpine $(\boldsymbol{e})$. Sleep is plotted as minutes of sleep per sliding $30 \mathrm{~min}$ window across a $24 \mathrm{~h}$ period ( $12 \mathrm{~h}$ light, white bar; and $12 \mathrm{~h}$ dark, black bar) with averaged data from 4 female flies over $5 \mathrm{~d}$ of recording.

Importantly, inhibition of VMAT by drug treatment or genetic mutation does not render flies hypoactive, as measured by activity index (activity per waking minute) (Fig. 3b), indicating that the effect is specific for sleep.

We next asked whether inhibition of VMAT causes increased sleep depth in addition to increased sleep quantity. To assess sleep depth, we measured arousal threshold, in other words, the ability of the animal to wake up with sensory stimulation. We delivered weak, medium, and strong stimuli to flies at different times of the night and counted the number aroused in different fly lines. We found that a smaller percentage of the drug-treated iso31 and the $V M A T^{p 1}$ homozygous mutants awaken in response to these mechanical stimuli, compared with untreated iso31 flies (Fig. $3 c$ ). The contribution of genotype to arousability at all stimulus intensities was significant ( $p<0.0001$ by two-way ANOVA).

We also measured the effect of VMAT inhibition on the homeostatic rebound that follows a period of sleep deprivation. We 


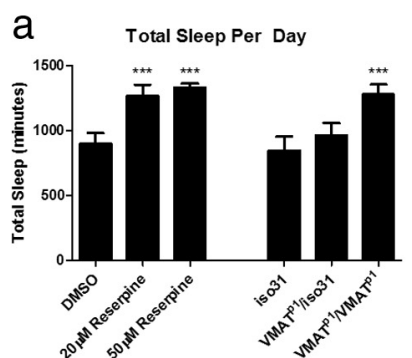

b

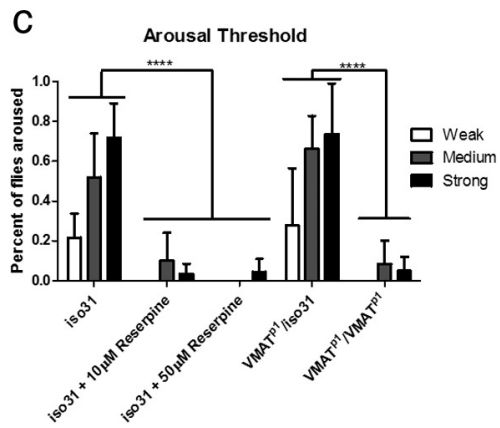

Df(2R)BSC306, which spans the VMAT locus (Fig. 4c). Together, these data indicate that VMAT mutants are resistant to reserpine, and the effect maps to the VMAT locus.

The sleep phenotype of VMAT mutants is background dependent

To determine whether the sleep phenotype of the VMAT ${ }^{p 1}$ mutation is independent of genetic background, we outcrossed the mutation for five generations into an iso31 background. The presence of the transposon was confirmed by PCR (Fig. 4b). Surprisingly, outcrossed VMAT mutant flies $\left(V M A T^{p 1}(5 x)\right.$ flies) slept for approximately the same number of minutes per day as the iso31 control flies $(846 \mathrm{~min} / \mathrm{d}$ for iso31 females vs $881 \mathrm{~min} / \mathrm{d}$ for $\operatorname{VMAT}^{p 1}(5 x) /$ $\operatorname{VMAT}^{p 1}(5 x)$; Fig. $\left.5 a, b\right)$. Despite the largely normal baseline sleep behavior, however, the $\operatorname{VMAT}^{p 1}(5 x)$ flies consistently exhibited decreased sleep latency, which means that they fell asleep more quickly than wild-type or heterozygous flies after lights out $(p=$ 0.035 by unpaired $t$ test with Welch's correction for unequal variances; Fig. 5c). Additionally, these mutant flies retained an increased arousal threshold after outcrossing (Fig. $5 d$ ), similar to the original mutant strain $(p=0.0100$ by two-way ANOVA for genotype contribution to arousability). Also, like the original mu-

Figure 3. Genetic ablation of VMAT alters sleep behavior. $\boldsymbol{a}$, Total minutes of sleep per $24 \mathrm{~h}$ day plotted for wild-type (iso31) flies fed $0.1 \%$ DMSO (vehicle control), $20 \mu \mathrm{m}$ reserpine, and $50 \mu \mathrm{m}$ reserpine, as well as for is031, VMAT ${ }^{p} /$ iso 31 , and $V M A T^{p 1} / V M A T^{p}$ fed sucrose/agar food. $n=8$ for each genotype/treatment. ${ }^{* * *} p<0.001 . \boldsymbol{b}$, Activity index (infrared beam crosses per waking minute) for these reserpine-treated and $V M A T^{p 1}$ mutant flies shows these flies are not hypoactive. $c$, The percentage of sleeping flies that were aroused from sleep by a weak (white bars), medium (gray bars), or strong (black bars) stimulus at ZT16, ZT18, or ZT20, respectively. Data are averaged from three separate experiments. ${ }^{* * *} p<0.0001$, the effect of treatment/genotype on arousability. ns, Not significant. $\boldsymbol{d}$, Flies were deprived of sleep during the second half of the night (ZT18-ZT24). Minutes of sleep lost during this period (white bar) are plotted as a negative number, and rebound sleep was measured during the first 3 (light gray bar), 6 (gray bar), and $12 \mathrm{~h}$ (black bar) the following morning. ${ }^{* *} p<0.01$, the amount of sleep lost compared with is 031 controls. ${ }^{* * *} p<0.001$, the amount of sleep lost compared with is 031 controls. $n=16$ for each genotype/treatment.

deprived flies of sleep during the second half of the night and assayed rebound sleep the following morning. Drug-treated and homozygous mutant flies were less effectively deprived than wildtype and heterozygous flies $(p=0.00322$ and $p=0.000185$ for iso31 vs $V M A T^{p 1} / V M A T^{p 1}$ and reserpine-treated flies, respectively, by one-way ANOVA with Tukey's post hoc), further supporting the idea that sleep depth is increased by inhibition of VMAT. Mutant and drug-treated flies experienced slightly less rebound sleep as well, although this difference did not reach significance because of large interindividual variance (Fig. $3 d$ ). The apparent reduction in sleep rebound by these flies is likely the result of the relatively ineffective deprivation and already elevated baseline sleep levels.

\section{Effects of reserpine on sleep map to the VMAT gene}

In VMAT ${ }^{p 1}$ flies, the VMAT gene is disrupted by insertion of a PLacW transposon in the fifth exon of the VMAT gene (Bellen et al., 2011). To confirm the presence of this transposon, we genotyped $V M A T^{p l}$ flies by PCR. The wild-type VMAT allele, detected using primers specific for the VMAT genomic sequence, was amplified from iso31 flies and from flies heterozygous for the mutation, but not from homozygous mutants. Heterozygous and homozygous mutants were positive for the mutant VMAT allele, which was detected using one VMAT primer and one transposon-specific primer (Fig. $4 a, b$ ).

If effects of reserpine on sleep are mediated through inhibition of VMAT, then VMAT mutants should be resistant to reserpine. Indeed, the $V M A T^{p 1}$ mutant does not show a further sleep increase after reserpine administration (Fig. 4c). Additionally, reserpine resistance of the VMAT ${ }^{p l}$ mutant is not complemented by the deficiency tant, the $\operatorname{VMAT}^{p 1}(5 x)$ mutants were less efficiently sleepdeprived ( $p=0.0003$ by one-way ANOVA) but showed a rebound the following morning proportional to the sleep lost during deprivation (Fig. 5e).

Importantly, $\operatorname{VMAT}^{p 1}(5 x)$ mutants were still resistant to the sleep-promoting effects of reserpine (Fig. $5 b$ ). Because these mutants have normal baseline sleep, the lack of a response to reserpine cannot be the result of a "ceiling" effect. These data establish that VMAT is required for effects of reserpine on sleep.

\section{Effects of reserpine on mutants of different monoaminergic systems}

VMAT is a transporter protein that packages all monoaminergic neurotransmitters into presynaptic vesicles. To determine which of the monoamines is responsible for the sleep phenotype produced by reserpine, we fed reserpine to mutants deficient for the various monoamines. Mutants deficient in the synthesis of dopamine $\left(p l e^{t s}\right)$, serotonin $\left(T r H^{c 01440}\right)$, octopamine $\left(\mathrm{TbH}^{\mathrm{nm} 18}\right)$, histamine $\left(H d c^{\mathrm{MBO} 0212}\right)$, and the amino acidderived neurotransmitter GABA ( $\mathrm{Gadl}^{\mathrm{f00602}}$ ) all responded to reserpine (Fig. 6). Because dopamine synthesis is required during development, the only viable mutant (ple) is temperaturesensitive, and so the drug treatment and sleep behavior for this mutant were measured at the restrictive temperature of $29^{\circ} \mathrm{C}$. Reserpine increased sleep significantly for all of the neurotransmitter mutants (comparing total sleep of DMSO controls with 10 $\mu \mathrm{M}$ reserpine-treated flies of the following genotypes, $p=$ 0.000139 for iso31, $p=0.00355 p l e^{t s}$ at $29^{\circ} \mathrm{C}, p=0.000144$ for $\mathrm{TrH}^{\mathrm{col} 1440}, p=0.000137$ for $T b H^{n m 18}, p=0.000137$ for $H d c^{M B 07212}$, and $p=0.000151$ for Gad1 $1^{f 0062}$ by two-way ANOVA 
a
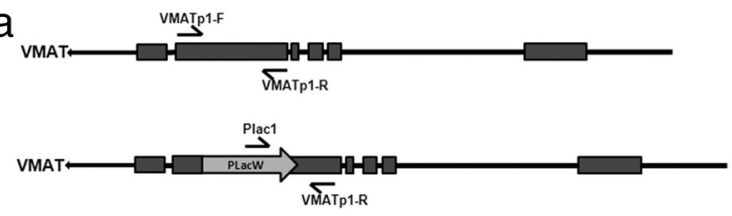

b
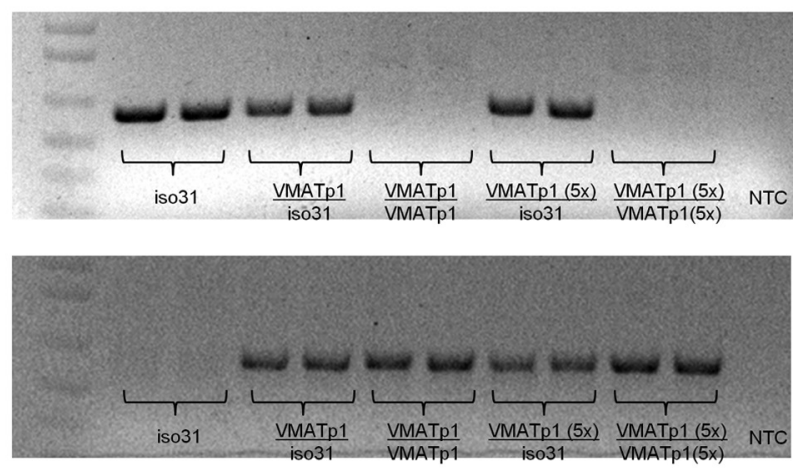

C

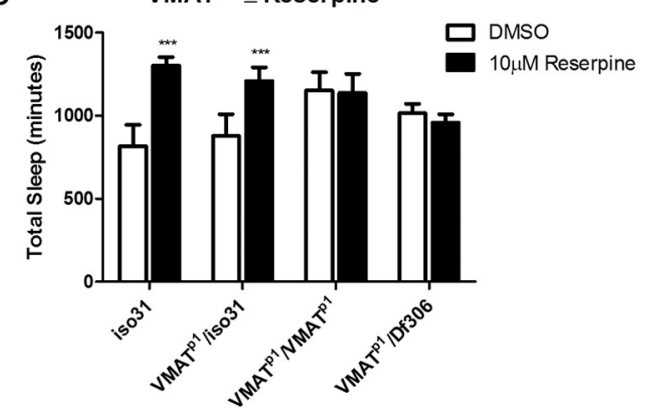

Figure 4. Effects of reserpine on sleep map to the VMAT gene. $\boldsymbol{a}$, The VMAT gene, with dark gray boxes representing exons, showing locations of $P C R$ primers used to genotype the wildtype VMAT (top) and P-element-containing mutant VMAT ${ }^{p 1}$ (bottom) alleles. $\boldsymbol{b}, \mathrm{PCR}$ amplifies the wild-type VMAT allele (top) in iso31 and VMAT ${ }^{p 1} /$ iso31 heterozygous flies and the VMAT ${ }^{p 1}$ mutant allele (bottom) in heterozygous and homozygous VMAT ${ }^{p 1}$ mutant flies. c, Total minutes of sleep per $24 \mathrm{~h}$ day for wild-type, heterozygous mutant, homozygous mutant, and mutant/ deficiency trans-heterozygotes fed 0.1\% DMSO (white bars) or $10 \mu \mathrm{m}$ reserpine (black bars). ${ }^{* * *} p<0.001 . n=16$ for each genotype/treatment.

with Bonferroni multiple comparisons), indicating that no single neurotransmitter system is required for sleep-promoting effects of reserpine. VMAT inhibition likely increases sleep by interfering with the signaling from more than one neurotransmitter system simultaneously.

\section{Discussion}

Genetic screens for sleep phenotypes have led to the isolation of a few mutants, including Shaker (Cirelli et al., 2005), sleepless (Koh et al., 2008), insomniac (Stavropoulos and Young, 2011), and cyclinA1 (Rogulja and Young, 2012). Other mutations that cause reduced sleep were identified by chance, including fumin (Kume et al., 2005) and several mutations in the calcineurin signaling pathway (Nakai et al., 2011), or through assays of candidate genes (Yuan et al., 2006; Crocker and Sehgal, 2008, 2010; Sehgal and Mignot, 2011). Although these studies give valuable insight into molecular underpinnings of sleep behavior, they do not paint a complete picture of the molecular machinery of sleep regulation.

We note that traditional genetic screens may be limited in their ability to uncover molecules that regulate behavior because of factors, such as redundancy, lethality, developmental compensation, and developmental defects, which may mask or conflate adult phenotypes. The study of sleep is particularly susceptible to these limi- tations, as long-term sleep deprivation leads to death (Rechtschaffen et al., 1983; Shaw et al., 2009). In addition, sleep-regulating genes tend to also be required for other functions. One way to bypass the limitations intrinsic to traditional genetic screens is to use adultspecific manipulations. We asked whether we could use a smallmolecule screen to discover new sleep-modulating proteins.

Small-molecule screens in whole animals are rare, especially when measuring a behavioral output. A small-molecule screen for aberrant sleep behavior in zebrafish assayed larvae, through automated methods, for effects of almost 4000 drugs (Rihel et al., 2010). Conducting a drug screen in Drosophila, although more labor-intensive, was important to find new sleep-modulating molecular targets in a well-established sleep model. Additionally, an unparalleled genetic toolkit is available in Drosophila for confirming and elaborating on drug screen findings.

In the screen reported here, we searched for drugs that dosedependently and reproducibly affected sleep behavior in both male and female adult flies. Two of the compounds that met these strict criteria are dopamine receptor agonists. Other drugs that increase dopamine signaling, such as methamphetamine and cocaine, are known to increase arousal in humans and model organisms, including Drosophila. Inhibition of dopamine biosynthesis biochemically (Andretic et al., 2005) and genetically (Riemensperger et al., 2011) has the opposite effect, increasing sleep amount in the fly. Also, the dopamine type 1 receptor, DopR, promotes arousal at appropriate times in the circadian sleep/wake cycle (Lebestky et al., 2009). The identification of small molecules targeting dopaminergic signaling validates the power of the drug screen to identify sleep-regulatory pathways.

Another sleep-inhibiting molecule identified in the screen was methylxanthine, which is a caffeine analog. Caffeine is well known as a robust wake-promoting stimulant. Although the target of its action in Drosophila is still unclear, its effects on behavior are similar to those in mammals/humans (Wu et al., 2008). As in the case of the molecules that affect dopaminergic signaling, the identification of a caffeine analog speaks to the efficacy of the small-molecule screen reported here. This screen also identified an atypical antipsychotic, paliperidone, as a sleep-inhibiting molecule. The target of this antipsychotic is not known, but its effect on sleep supports reports of links between sleep and affective disorders (Wulff et al., 2010).

Surprisingly, only one drug from the screen was found to increase sleep: reserpine. Although reserpine, which is typically used to treat hypertension and is also indicated as an antipsychotic, was shown many years ago to have a tranquilizing effect, it has not been mechanistically linked to sleep (Monroe et al., 1955; Steiner et al., 1963). We now have the genetic tools to understand the nature of this effect in a controlled and systematic manner and its implications for the normal regulation of sleep and wake. Reserpine inhibits the function of the VMAT, a transmembrane protein that transports monoaminergic neurotransmitters into presynaptic vesicles to prepare them for release. Vertebrates have two VMAT genes, VMAT1 and VMAT2, whereas flies have only one.

One common caveat in pharmacological studies is the possibility of off-target effects. We show that a VMAT ${ }^{\text {pl-null }}$ mutant (Simon et al., 2009) has increased sleep. More importantly, this mutant is resistant to the effects of reserpine, indicating that the long-sleeping phenotype is not the result of off-target effects. An apparent increase in sleep can sometimes result from sickness or physical impairment. However, despite sleeping significantly more, the reserpine-fed and VMAT ${ }^{p l}$ mutant flies do not have a decreased activity index. In addition, outcrossed VMAT mutants, which have normal levels of baseline sleep, are unresponsive to 

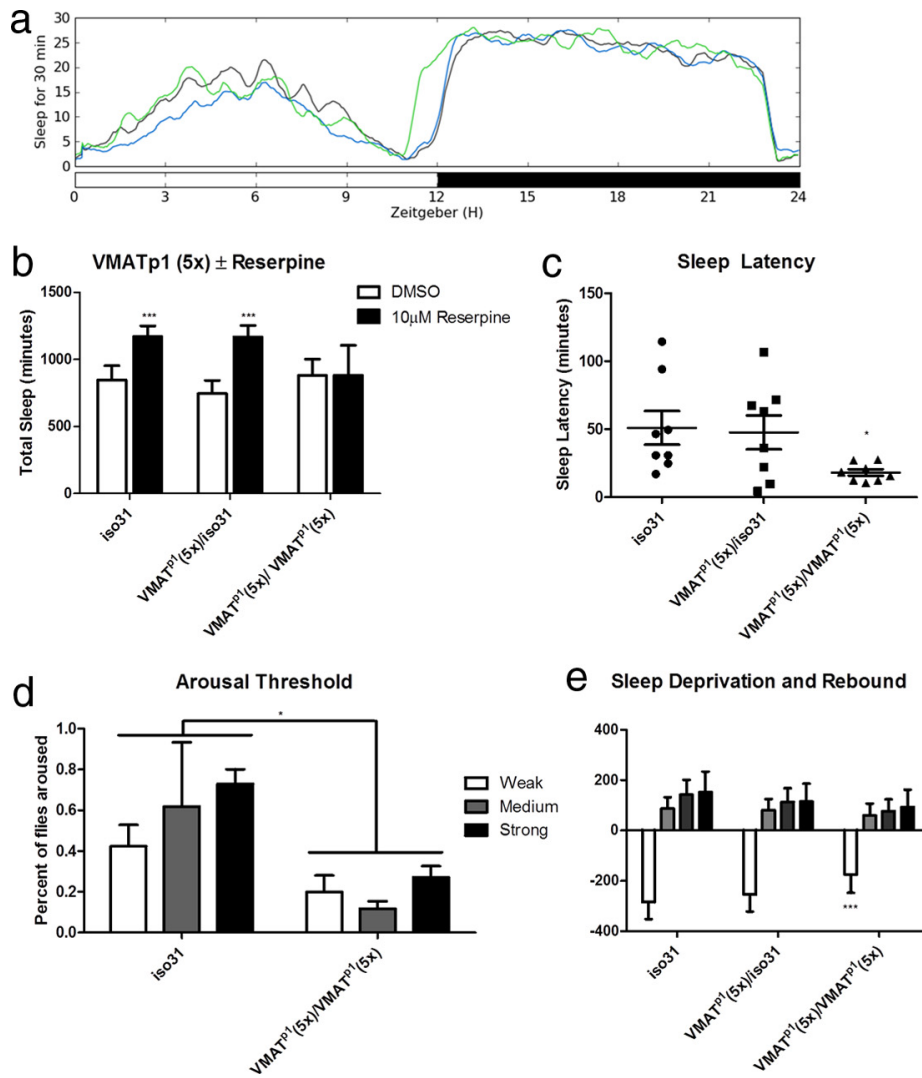

Sleep Deprivation and Rebound

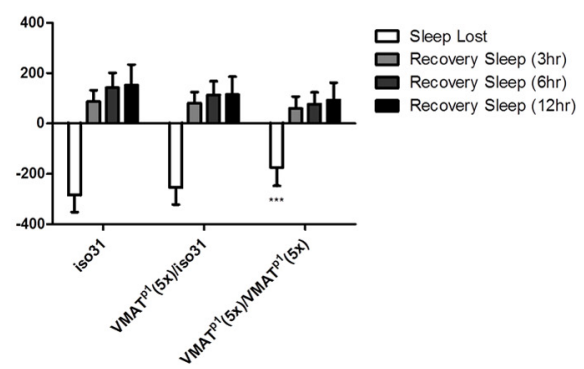

Figure 5. The sleep phenotype of VMAT mutants is background dependent. $\boldsymbol{a}$, Sleep profile for iso31 (black line), VMAT ${ }^{p 1}(5 x) /$ is 031 (blue line), and $V M A T^{p 1}(5 x) / V M A T^{p 1}(5 x)$ (green line) outcrossed mutant flies. Sleep is plotted as minutes of sleep per sliding $30 \mathrm{~min}$ window across a $24 \mathrm{~h}$ period ( $12 \mathrm{~h}$ light, white bar; and $12 \mathrm{~h}$ dark, black bar) with averaged data from 16 female flies over $5 \mathrm{~d}$ of recording. $\boldsymbol{b}$, Total sleep per $24 \mathrm{~h}$ period quantified for these flies fed $0.1 \%$ DMSO (white bars) or $10 \mu \mathrm{m}$ reserpine (black bars). ${ }^{* * *} p<0.001$. c, Scatter plot of latency to sleep, or minutes between lights off and the first sleep bout. Horizontal line corresponds to group mean. ${ }^{*} p<0.05$. $\boldsymbol{d}$, The percentage of sleeping flies that were aroused from sleep by a weak (white bars), medium (gray bars), or strong (black bars) stimulus at ZT16, ZT18, or ZT20, respectively. Data are averaged from three independent experiments. ${ }^{*} p<0.05$, the effect of genotype on arousability. $e$, Flies were deprived of sleep during the second half of the night (ZT18-ZT24). Minutes of sleep lost during this period (white bar) are plotted as negative numbers, and rebound sleep was measured during the first 3 (light gray bar), 6 (gray bar), and $12 \mathrm{~h}$ (black bar) the following morning. ${ }^{* *} p<0.001$, the amount of sleep lost compared with iso31 controls. $n=16$ for each genotype.

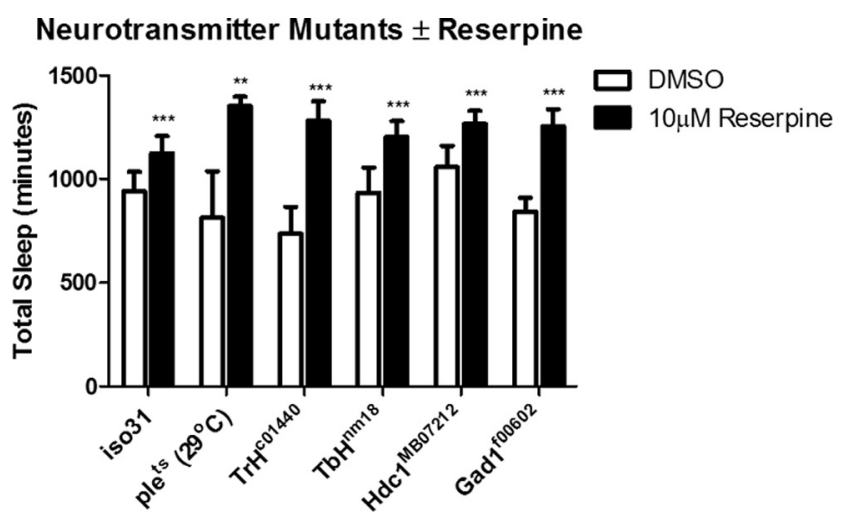

Figure 6. Effects of reserpine on mutants of different monoaminergic systems. Total sleep per $24 \mathrm{~h}$ period plotted for mutants defective in the synthesis of the neurotransmitters dopamine ( $p l e^{t s}$ at $29^{\circ} \mathrm{C}$ ), serotonin $\left(\mathrm{TrH}^{\mathrm{ro1449}}\right)$, octopamine $\left(\mathrm{TbH}^{\mathrm{nm} 18}\right)$, histamine $\left(\mathrm{HdC}^{\mathrm{MBO} 0212}\right.$ ), and GABA (Gad7 f00602), fed $0.1 \%$ DMSO (white bars) or $10 \mu$ m reserpine (blackbars). ${ }^{* *} p<0.01 .{ }^{* * *} p<0.001$.

reserpine. Together, these data show that reserpine specifically increases sleep by inhibiting VMAT.

Alterations in sleep duration are often accompanied by changes in sleep depth, as measured by arousal threshold. Previ- ous studies have shown that shortsleeping mutants tend to have decreased arousal thresholds during normal sleep (Koh et al., 2008). Interestingly, the longsleeping $V M A T^{p 1}$ mutant has an increased arousal threshold, suggesting that they sleep more deeply (Andretic and Shaw, 2005). In general, loss of VMAT appears to increase sleep drive or decrease the ability to maintain wakefulness, as demonstrated also by the increased latency to sleep in flies carrying a fivegeneration outcrossed VMAT $T^{p 1}$ allele. These outcrossed flies no longer have increased daily sleep, but they also display an increased arousal threshold. On the other hand, VMAT $T^{p 1}$ flies have a normal rebound after deprivation, supporting the idea that the response to sleep deprivation is less tightly correlated with other measures of sleep.

The less severe phenotype of the outcrossed allele is consistent with other studies that have noted the importance of genetic background in animal behaviors, including sleep (Zimmerman et al., 2012). In an iso31 background, inhibition of VMAT throughout development with the $V M A T^{p 1}$ mutation does not alter daily sleep, but sleep is increased sleep when VMAT is inhibited acutely in adults. Thus, developmental compensation mechanisms may account for the discrepancy between the sleep phenotype of the drug-fed and mutant flies in the iso31 genetic background. The original mutant background likely confers less developmental compensation, as these flies have the same longsleeping phenotype as the drug-fed flies.

VMAT plays a presynaptic role in signaling by many different neurotransmitters, including the monoamine neurotransmitters dopamine, serotonin, histamine, and octopamine. Additionally, recent evidence suggests that VMAT transports the amino acid neurotransmitter GABA (Tritsch, 2012). Many of these neurotransmitters, including dopamine (Andretic et al., 2005; Kume et al., 2005), octopamine (Crocker and Sehgal, 2008), serotonin (Yuan et al., 2006), and GABA (Agosto et al., 2008), have been independently implicated in regulating sleep behavior. We found that mutants deficient for each of these neurotransmitters displayed increased sleep after reserpine feeding, suggesting that no single neurotransmitter system accounts for the impact of VMAT inhibition on sleep. Similarly, Chen et al. (2013) found that circadian rhythms are perturbed in $V M A T^{p 1}$ mutants, and rescue of this phenotype requires VMAT in multiple neuronal populations. In mammals as well, Coulter et al. (1971) demonstrated that the effect of reserpine on sleep cannot be attributed to reductions in serotonin or norepinephrine. Our findings contribute to a picture of sleep regulation driven by a robust network of neurotransmission that requires VMAT in multiple neuronal populations.

Altered VMAT function has previously been studied in the context of many neuropsychiatric and neurological diseases, including depression, bipolar disorder, schizophrenia, and Parkinson's disease 
(Wimalasena, 2011). These diseases are accompanied by an increased prevalence of sleep perturbations, although these have not yet been linked to VMAT. Understanding the role of VMAT in sleep may elucidate the pathophysiology of sleep perturbations in the disorders noted here, as well as the natural regulation of sleep in healthy flies and humans.

The potential for screens in Drosophila to identify drugs for human use is high. Although numerous side effects make reserpine suboptimal as a treatment, more specific inhibitors of VMAT-2 may be tolerated better by patients and improve their use as a sleep aid. The screen reported here identified a single sleep-promoting drug, but expanded screens could identify many more potential pharmacotherapies. We used a drug library with known biological targets, which may have biased the findings toward well-studied pathways. Additionally, a low concentration of drug was used to reduce lethality, meaning that only drugs with the strongest impacts on sleep were found. Now that the utility of these screens has been proven, larger and more aggressive screens can be used to identify other novel modulators of behavior.

In conclusion, we used a small-molecule screen to discover regulators of sleep phenotype. Using a genetic approach to confirm one of these drugs, we found that VMAT is required to establish normal sleep duration and arousal state, presumably by regulating transmission of several neurotransmitters. The role of genetic background in the expressivity of the VMAT phenotype highlights the strong effect of developmental compensation on behaviors, such as sleep, and the importance of targeting pathways acutely in adults to look at adult behavior. Small-molecule screens in live animals provide a powerful tool for dissecting molecular mechanisms of adult behavior.

\section{References}

Agosto J, Choi JC, Parisky KM, Stilwell G, Rosbash M, Griffith LC (2008) Modulation of GABAA receptor desensitization uncouples sleep onset and maintenance in Drosophila. Nat Neurosci 11:354-359. CrossRef Medline

Allada R, Siegel JM (2008) Unearthing the phylogenetic roots of sleep. Curr Biol R670-R679.

Andretic R, Shaw PJ (2005) Essentials of sleep recordings in Drosophila: moving beyond sleep time. Methods Enzymol 393:759-772. CrossRef Medline

Andretic R, van Swinderen B, Greenspan RJ (2005) Dopaminergic modulation of arousal in Drosophila. Curr Biol 15:1165-1175. CrossRef Medline

Bellen HJ, Levis RW, He Y, Carlson JW, Evans-Holm M, Bae E, Kim J, Metaxakis A, Savakis C, Schulze KL, Hoskins RA, Spradling AC (2011) The Drosophila gene disruption project: progress using transposons with distinctive site specificities. Genetics 188:731-743. CrossRef Medline

Bushey D, Cirelli C (2011) From genetics to structure to function: exploring sleep in Drosophila. Int Rev Neurobiol 99:213-244. CrossRef Medline

Chen A, Ng F, Lebestky T, Grygoruk A, Djapri C, Lawal HO, Zaveri HA, Mehanzel F, Najibi R, Seidman G, Murphy NP, Kelly RL, Ackerson LC, Maidment NT, Jackson FR, Krantz DE (2013) Dispensable, redundant, complementary, and cooperative roles of dopamine, octopamine, and serotonin in Drosophila melanogaster. Genetics 193:159-176. CrossRef Medline

Cirelli C, Bushey D, Hill S, Huber R, Kreber R, Ganetzky B, Tononi G (2005) Reduced sleep in Drosophila Shaker mutants. Nature 434:1087-1092. CrossRef Medline

Coulter JD, Lester BK, Williams HL (1971) Reserpine and sleep. Psychopharmacologia 19:134-147. CrossRef Medline

Crocker A, Sehgal A (2008) Octopamine regulates sleep in drosophila through protein kinase A-dependent mechanisms. J Neurosci 28:9377-9385. CrossRef Medline

Crocker A, Sehgal A (2010) Genetic analysis of sleep. Genes Dev 24:12201235. CrossRef Medline

Gilestro GF, Cirelli C (2009) pySolo: a complete suite for sleep analysis in Drosophila. Bioinformatics 25:1466-1467. CrossRef Medline

Hendricks JC, Finn SM, Panckeri KA, Chavkin J, Williams JA, Sehgal A, Pack
AI (2000) Rest in Drosophila is a sleep-like state. Neuron 25:129-138. CrossRef Medline

Huber R, Hill SL, Holladay C, Biesiadecki M, Tononi G, Cirelli C (2004) Sleep homeostasis in Drosophila melanogaster. Sleep 27:628-639. Medline

Koh K, Joiner WJ, Wu MN, Yue Z, Smith CJ, Sehgal A (2008) Identification of SLEEPLESS, a sleep-promoting factor. Science 321:372-376. CrossRef Medline

Kume K, Kume S, Park SK, Hirsh J, Jackson FR (2005) Dopamine is a regulator of arousal in the fruit fly. J Neurosci 25:7377-7384. CrossRef Medline

Lebestky T, Chang JS, Dankert H, Zelnik L, Kim YC, Han KA, Wolf FW, Perona P, Anderson DJ (2009) Two different forms of arousal in Drosophila are oppositely regulated by the dopamine D1 receptor ortholog DopR via distinct neural circuits. Neuron 64:522-536. CrossRef Medline

Mackiewicz M, Naidoo N, Zimmerman JE, Pack AI (2008) Molecular mechanisms of sleep and wakefulness. Ann N Y Acad Sci 1129:335-349. CrossRef Medline

Monroe RR, Heath RG, Mickle WA, Miller W (1955) A comparison of cortical and subcortical brain waves in normal, barbiturate, reserpine, and chlorpromazine sleep. Ann N Y Acad Sci 61:56-71. CrossRef Medline

Nakai Y, Horiuchi J, Tsuda M, Takeo S, Akahori S, Matsuo T, Kume K, Aigaki T (2011) Calcineurin and its regulator sra/DSCR1 are essential for sleep in Drosophila. J Neurosci 31:12759-12766. CrossRef Medline

Pendleton RG, Rasheed A, Sardina T, Tully T, Hillman R (2002) Effects of tyrosine hydroxylase mutants on locomotor activity in Drosophila: a study in functional genomics. Behav Genet 32:89-94. CrossRef Medline

Raizen DM, Zimmerman JE, Maycock MH, Ta UD, You YJ, Sundaram MV, Pack AI (2008) Lethargus is a Caenorhabditis elegans sleep-like state. Nature 451:569-572. CrossRef Medline

Rechtschaffen A, Gilliland MA, Bergmann BM, Winter JB (1983) Physiological correlates of prolonged sleep deprivation in rats. Science 221:182184. CrossRef Medline

Riemensperger T, Isabel G, Coulom H, Neuser K, Seugnet L, Kume K, IchéTorres M, Cassar M, Strauss R, Preat T, Hirsh J, Birman S (2011) Behavioral consequences of dopamine deficiency in the Drosophila central nervous system. Proc Natl Acad Sci U S A 108:834-839. CrossRef Medline

Rihel J, Prober DA, Arvanites A, Lam K, Zimmerman S, Jang S, Haggarty SJ, Kokel D, Rubin LL, Peterson RT, Schier AF (2010) Zebrafish behavioral profiling links drugs to biological targets and rest/wake regulation. Science 327:348-351. CrossRef Medline

Rogulja D, Young MW (2012) Control of sleep by cyclin A and its regulator. Science 335:1617-1621. CrossRef Medline

Ryder E, Blows F, Ashburner M, Bautista-Llacer R, Coulson D, Drummond J, Webster J, Gubb D, Gunton N, Johnson G, O'Kane CJ, Huen D, Sharma P, Asztalos Z, Baisch H, Schulze J, Kube M, Kittlaus K, Reuter G, Maroy P, et al. (2004) The DrosDel collection: a set of P-element insertions for generating custom chromosomal aberrations in Drosophila melanogaster. Genetics 167:797-813. CrossRef Medline

Sehgal A, Mignot E (2011) Genetics of sleep and sleep disorders. Cell 146: 194-207. CrossRef Medline

Shaw PJ, Cirelli C, Greenspan RJ, Tononi G (2000) Correlates of sleep and waking in Drosophila melanogaster. Science 287:1834-1837. CrossRef Medline

Shaw PJ, Tononi G, Greenspan RJ, Robinson DF (2002) Stress response genes protect against lethal effects of sleep deprivation in Drosophila. Nature 417:287-291. CrossRef Medline

Simon AF, Daniels R, Romero-Calderón R, Grygoruk A, Chang HY, Najibi R, Shamouelian D, Salazar E, Solomon M, Ackerson LC, Maidment NT, Diantonio A, Krantz DE (2009) Drosophila vesicular monoamine transporter mutants can adapt to reduced or eliminated vesicular stores of dopamine and serotonin. Genetics 181:525-541. CrossRef Medline

Stavropoulos N, Young MW (2011) insomniac and Cullin-3 regulate sleep and wakefulness in Drosophila. Neuron 72:964-976. CrossRef Medline

Steiner WG, Pscheidt GR, Himwich HE (1963) Influence of methodology on electroencephalographic sleep and arousal: studies with reserpine and etryptamine in rabbits. Science 141:53-55. CrossRef Medline

Tritsch NX, Ding JB, Sabatini BL (2012) Dopaminergic neurons inhibit striatal output through non-canonical release of GABA. Nature 490:262-266. CrossRef Medline

Wimalasena K (2011) Vesicular monoamine transporters:structure-func- 
tion, pharmacology, and medicinal chemistry. Med Res Rev 31:483-519. CrossRef Medline

Wu MN, Koh K, Yue Z, Joiner WJ, Sehgal A (2008) A genetic screen for sleep and circadian mutants reveals mechanisms underlying regulation of sleep in Drosophila. Sleep 31:465-472. Medline

Wulff K, Gatti S, Wettstein JG, Foster RG (2010) Sleep and circadian rhythm disruption in psychiatric and neurodegenerative disease. Nat Rev Neurosci 11:589-599. CrossRef Medline
Yuan Q, Joiner WJ, Sehgal A (2006) A sleep-promoting role for the Drosophila serotonin receptor 1A. Curr Biol 16:1051-1062. CrossRef Medline

Zimmerman JE, Chan MT, Jackson N, Maislin G, Pack AI (2012) Genetic background has a major impact on differences in sleep resulting from environmental influences in Drosophila. Sleep 35:545-557. CrossRef Medline 